\title{
Espondilodiscite Brucélica: Casuística dos Últimos 25 Anos
}

\author{
Brucellar Spondylodiscitis: Case Series of the Last 25 Years
}

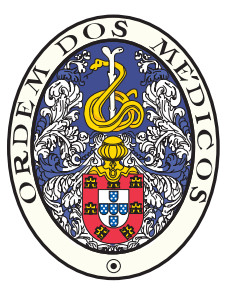

\author{
Ana LEBRE ${ }^{1}$, Jorge VELEZ ${ }^{2}$, Diana SEIXAS ${ }^{1}$, Eduardo RABADÃO ${ }^{1}$, Joaquim OLIVEIRA ${ }^{1}$, J. SARAIVA DA CUNHA ${ }^{1}$, A. $^{2}$ \\ Meliço SILVESTRE ${ }^{1}$ \\ Acta Med Port 2014 Mar-Apr;27(2):204-210
}

\section{RESUMO}

Introdução: A brucelose é uma zoonose endémica em Portugal, sendo a espondilodiscite brucélica uma das manifestações focais mais frequentes. Pode provocar sequelas graves, apesar da terapêutica dirigida.

Material e Métodos: Estudo retrospectivo dos processos dos doentes com espondilodiscite brucélica, internados no Serviço de Doenças Infecciosas do Centro Hospitalar e Universitário de Coimbra, num período de 25 anos (1988-2012).

Resultados: Foram identificados 54 doentes, $55,6 \%$ do sexo masculino, com idade média de 54,8 anos. Em $81,5 \%$ identificou-se contexto epidemiológico, maioritariamente contacto com gado ovino e caprino. A duração da sintomatologia prévia ao diagnóstico foi de 5,5 meses. Os sinais e sintomas mais frequentes foram: dor $(98,1 \%)$, febre $(46,3 \%)$ e défices neurológicos $(25,9 \%)$. A ressonância magnética nuclear da coluna foi o exame imagiológico mais usado $(77,8 \%)$ evidenciando abcessos em $29,6 \%$ dos doentes. A localização lombar predominou (77,7\%). O diagnóstico etiológico foi confirmado em 47 doentes (87,0\%): microbiologicamente (3 doentes), serologicamente (32 doentes) ou por ambos (12 doentes). As associações de doxiciclina com rifampicina (64,8\%), ou estreptomicina $(24,1 \%)$ foram as mais utilizadas, com duração média de 4,4 meses de tratamento. Um doente teve indicação cirúrgica para drenar abcesso. A evolução foi maioritariamente favorável (92,6\%), sem óbitos.

Discussão: A investigação de contexto epidemiológico revelou ser uma peça importante na suspeita do diagnóstico. O tratamento da brucelose osteoarticular ainda é controverso.

Conclusões: A espondilodiscite brucélica deve ser considerada no diagnóstico diferencial dos doentes com lombalgia, mesmo na ausência de febre, particularmente em regiões onde a doença é endémica. O esquema antibiótico, sua duração e a necessidade de cirurgia deverão ser individualizados, com vista a um melhor prognóstico. O número de casos tem diminuído ao longo dos anos, facto relacionado com melhor controlo da endemia animal.

Palavras-chave: Brucella; Brucelose; Discite; Osteomielite; Portugal.

\section{ABSTRACT}

Introduction: Brucellosis is an endemic zoonosis in Portugal. Brucellar spondylodiscitis is one of the most frequent focal manifestations which may cause severe sequelae despite appropriate therapy.

Material and Methods: Retrospective study of patients with diagnosis of brucellar spondylodiscitis admitted to the Infectious Diseases Department of Centro Hospitalar e Universitário de Coimbra, over a 25-year period (1988-2012).

Results: We identified 54 patients, $55.6 \%$ male, mean age of 54.8 years. In $81.5 \%$ an epidemiological context was identified, mostly contact with sheep and goats. The duration of symptoms prior to diagnosis was 5.5 months. The most common signs and symptoms were pain (98.1\%), fever (46.3\%) and neurological deficits $(25.9 \%)$. Spinal magnetic resonance imaging was the most used imaging method $(77.8 \%)$ showing abscesses in $29.6 \%$ of patients. Lumbar location predominated $(77.7 \%)$. Diagnosis was attained in 47 patients (87.0\%): positive blood cultures (3 patients), positive serology (32 patients) or by both methods (12 patients). Combined regimens of doxycycline and rifampicin (64.8\%), or streptomycin $(24.1 \%)$ were most used, for an average duration of 4.4 months. A patient was referred for surgery for abscess drainage. Evolution was mostly favorable (92.6\%), no deaths occurring.

Discussion: Research of the epidemiologic context turned out to be a major key leading to the diagnosis. Treatment of osteoarticular brucellosis is still controversial.

Conclusions: Brucellar spondylodiscitis should be considered in the differential diagnosis of patients with low back pain, even in the absence of fever, particularly in regions where the disease is endemic. Antibiotic regimen, its' duration and the need for surgery should be individualized to achieve a better prognosis. Cases have declined over the years, a fact related to better control of animal endemic. Keywords: Brucella; Brucellosis; Discitis; Osteomyelitis; Portugal.

\section{INTRODUÇÃO}

A espondilodiscite é uma infecção do disco intervertebral e vértebra adjacente que normalmente ocorre por disseminação hematogénica. O Staphylococcus aureus é, globalmente, o gérmen mais frequentemente implicado, ${ }^{1}$ contudo nas regiões onde a brucelose é endémica, esta etiologia deve ser considerada no diagnóstico diferencial. ${ }^{2,3}$

A brucelose é a zoonose mais frequente a nível mun- dial, com mais de 500000 novos casos diagnosticados anualmente. ${ }^{4} \mathrm{~A}$ bacia do Mediterrâneo é considerada uma região endémica, mas no que concerne ao nosso país, tem-se assistido a um decréscimo do número de casos de brucelose humana notificados. ${ }^{5,6} \mathrm{~A}$ transmissão desta doença ocorre sobretudo por consumo de leite ou derivados não pasteurizados ou contacto directo com animais infectados.

1. Serviço de Doenças Infecciosas. Centro Hospitalar e Universitário de Coimbra. Coimbra. Portugal.

2. Serviço de Infecciologia. Centro Hospitalar do Baixo Vouga. Aveiro. Portugal.

Recebido: 17 de Junho de 2013 - Aceite: 15 de Outubro de 2013 | Copyright @ Ordem dos Médicos 2014 
O diagnóstico baseia-se no isolamento da Brucella spp. por cultura, habitualmente de sangue. Contudo, pela relativa lentidão deste método aliada a uma taxa de isolamento variável (dependente de duração de infecção e métodos usados), na maioria dos casos são usados métodos serológicos. A doença pode afectar qualquer órgão ou sistema, sendo a focalização osteoarticular a mais frequente, particularmente ao nível da coluna vertebral. ${ }^{7} \mathrm{~A}$ espondilodiscite brucélica (EB) é uma das manifestações mais graves da infecção, podendo subsistir sequelas graves apesar de terapêutica dirigida. ${ }^{8} \mathrm{O}$ esquema antibiótico mais eficaz e a duração do tratamento mais adequada ainda não estão claramente definidos, porém alguns estudos revelam superioridade da associação de doxiciclina com estreptomicina. ${ }^{7}$

Este estudo tem por objectivo analisar as características epidemiológicas, clínicas, diagnósticas e terapêuticas dos casos de EB diagnosticados no nosso Serviço, comparando os resultados com os dados disponíveis na literatura. Como existem poucas séries portuguesas sobre esta patologia, reveste-se de particular importância caracterizar melhor a realidade nacional.

\section{MATERIAL E MÉTODOS}

Foi realizada uma análise retrospectiva dos processos clínicos dos doentes internados no Serviço de Doenças Infecciosas dos Centro Hospitalar e Universitário de Coimbra (CHUC), entre 01 Janeiro de 1988 e 31 de Dezembro de 2012, com o diagnóstico de EB.

O diagnóstico de espondilodiscite foi estabelecido com base numa clínica sugestiva (dor no segmento da coluna envolvido e/ou défice neurológico de novo resultante de compressão medular ou radicular, com ou sem febre) associado a estudo imagiológico compatível com espondilodiscite.

O diagnóstico etiológico de brucelose foi considerado confirmado por identificação de Brucella spp. em hemocultura e/ou na demonstração de títulos significativos, acima do limiar de significância (1/160), de anticorpos específicos por seroaglutinação. O diagnóstico etiológico foi considerado presuntivo quando baseado em dados epidemiológicos, serológicos e imagiológicos sugestivos, associadamente a resposta favorável à terapêutica antibiótica dirigida.

Foram analisadas as seguintes variáveis: idade, sexo, história epidemiológica, sinais e sintomas predominantes, tempo de evolução dos sintomas, duração de internamento (nos doentes com múltiplos internamentos, a duração considerada foi a soma dos mesmos), ano de diagnóstico, estudos imagiológicos efectuados (ressonância magnética nuclear (RMN) e/ou tomografia computorizada), nível vertebral envolvido, presença de abcessos, dados microbiológicos e serológicos, tratamento efectuado e evolução. As radiografias simples da coluna foram excluídas do estudo, pois numa quantidade considerável de processos clínicos, porventura devido à sua antiguidade, não estavam disponíveis.

A evolução foi descrita como favorável quando ocorreu melhoria da dor e/ou défices neurológicos após terapêutica e desfavorável nos casos em que a clínica se manteve inalterada ou ocorreu agravamento.

\section{RESULTADOS}

Durante o período de 25 anos foram identificados 54 doentes com espondilodiscite brucélica, correspondendo a uma média de 2,2 casos por ano (Fig. 1).

A maioria $(55,6 \%)$ era do sexo masculino, com média de idade de 54,8 anos à data do diagnóstico. Em 44 $(81,5 \%)$ doentes foi identificado contexto epidemiológico, sendo o contacto com gado ovino e/ou caprino o mais referido (Tabela 1).

O tempo que mediou o início dos sintomas e o diagnóstico de espondilodiscite foi determinado em 39 doentes, com uma média de 5,5 \pm 7,4 meses [mín. 1 semana - máx. três anos] e mediana de três meses. Os principais sinais e sintomas referidos à data de admissão foram dor local, febre e défices neurológicos (Tabela 1), tendo o internamento uma duração média de $29 \pm 17,3$ dias [mín 1 - máx 69].

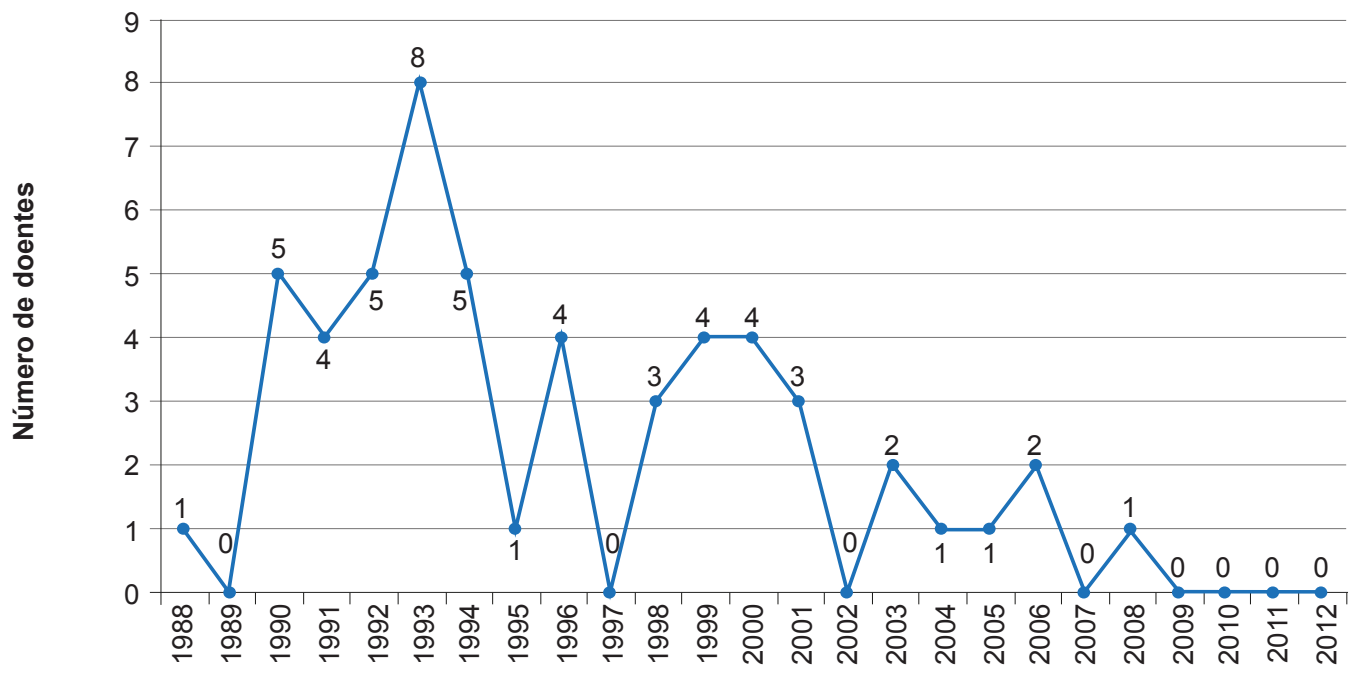

Ano de diagnóstico

Figura 1 - Distribuição anual dos doentes com espondilodiscite brucélica 
Relativamente ao estudo imagiológico, 42 (77,8\%) doentes realizaram RMN da coluna, com $97,6 \%$ (41/42) dos exames evidenciando alterações sugestivas de espondilodiscite. A tomografia computorizada da coluna foi realizada em 32 (59,3\%) doentes, apresentando em 81,3\% (26/32) achados sugestivos de espondilodiscite. Foram identificados abcessos em 16 (29,6\%) doentes: paravertebrais (oito doentes), do músculo psoas (seis doentes) e epidurais (cinco doentes). A distribuição anatómica das lesões está registada na Tabela 1, sendo o segmento lombar o mais frequentemente envolvido.

Todos os doentes efectuaram o teste de Rosa de Bengala, sendo positivo na totalidade dos mesmos. O diag- nóstico etiológico foi confirmado na maioria dos doentes $(87,0 \%)$, em 32 doentes por método serológico, em três por isolamento da bactéria em hemocultura e, em 12 casos, com ambos os exames positivos (Tabela 1).

Todos os doentes foram submetidos a antibioterapia dupla ou tripla (Tabela 2). As associações de doxiciclina com rifampicina $(64,8 \%)$ ou com estreptomicina $(24,1 \%)$ foram as mais utilizadas. A duração média do tratamento foi de 4,4 $\pm 2,3$ meses (mín. 1 - máx. 12). Um dos doentes foi submetido a cirurgia, para drenagem de abcesso paravertebral que condicionava compressão radicular.

Um resultado favorável, com melhoria ou resolução de queixas, foi observado em $92,6 \%$ dos doentes, enquanto

Tabela 1 - Características dos doentes com EB

Resultado

\section{Demográficas}

Idade, média \pm DP (min.- máx.)

$54,8 \pm 14,2(19-83)$

Sexo masculino, $n(\%)$

$30(55,6)$

\section{Epidemiológicas}

Contacto com gado ovino/caprino, $n(\%)$

Consumo de leite ou derivados não pasteurizados, $n(\%)$

História prévia de brucelose, $n(\%)$

$14(25,9)$

\section{Sintomas}

Dor local, $n(\%)$

Febre, $n(\%)$

Défices neurológicos, $n$ (\%)

\section{Nível vertebral das lesões}

Cervical, $n(\%)$

Dorsal, $n(\%)$

Dorso-lombar, $n$ (\%)

Lombar, $n$ (\%)

Lombo-sagrado, $n$ (\%)

\section{Dados serológicos e microbiológicos}

Teste de Rosa Bengala, $n$ (\%)

Teste de Wright, $n$ (\%)

$\begin{array}{lr}\text { Negativo } & 6(11,1) \\ 1 / 80 & 4(7,4) \\ 1 / 160 & 15(27,8) \\ 1 / 320 & 18(33,3) \\ 1 / 640 & 9(16,7) \\ 1 / 1280 & 2(3,7) \\ \text { Hemocultura positiva, } n(\%) & 15(27,8)\end{array}$


$\mathrm{Nr}$. de tratamentos

(\%)

$35(64,8)$

$13(24,1)$

$3(5,6)$

Doxiciclina + Gentamicina

$1(1,8)$
$4,4(1,4)$

$3,5(0,5)$

Duração média em meses

(DP)

$4,4(2,3)$

$5,0(0)$

$5,0(0)$
3,7\% não apresentaram melhora clínica após conclusão da terapêutica (um doente, com doença a nível L2-L5, manteve parestesias nos membros inferiores e outro, com lesão de localização L5-S1, lombalgia de caracteristicas mecânicas incapacitante). Os restantes 3,7\% foram transferidos e nenhum doente faleceu.

\section{DISCUSSÃO}

A brucelose foi, até há alguns anos atrás, uma das zoonoses com maior incidência a nível nacional, estando abrangida pelo sistema de doenças de declaração obrigatória. A evolução do número de casos notificados aponta para um decréscimo da endemia: segundo os dados da Direcção Geral de Saúde, desde 1994 até 2008 o número de casos de brucelose declarados diminuiu, de uma forma consistente, de 1240 até 56 casos. .,6 $^{5}$

A brucelose é uma infecção sistémica, na qual a maioria dos orgãos e sistemas podem ser afectados. As complicações osteoarticulares ocorrem em $20-40 \%$ dos casos de brucelose. ${ }^{7}$ Em três relevantes estudos sobre brucelose, a percentagem de espondilodiscite encontrada variou entre $9,7 \%$ e $12,3 \% .^{9-11}$

Os autores apresentam os 54 casos de espondilodiscite brucélica diagnosticados nos últimos 25 anos (1988-2012) no Serviço de Doenças Infecciosas de um hospital terciário da zona centro do país, dando continuidade à publicação que apresentou os resultados dos primeiros quatro anos desta casuística (1988-1991). ${ }^{12}$ A distribuição do número de casos ao longo do período estudado foi desigual, com a maioria dos casos a serem diagnosticados na década de 90 e ausência de casos de EB nos últimos cinco anos. Estes dados são concordantes com o decréscimo de casos de brucelose declarados na zona centro do país. ${ }^{5,6}$ Relativamente à prevalência de género, detectamos uma discreta predominância do sexo masculino (55,6\%), um valor inferior ao encontrado em algumas séries, ${ }^{7,9,13}$ mas similar a outras. ${ }^{14} \mathrm{De}$ acordo com a literatura ${ }^{9,15}$ os doentes com brucelose que desenvolvem EB são tendencialmente mais velhos, com idade entre os 50-60 anos, o que coincide com os nossos resultados (idade média de 54,8 \pm 14,2 anos).

A investigação de contexto epidemiológico revelou ser uma peça importante na suspeita do diagnóstico, com ape- nas $18,5 \%$ dos doentes não apresentando epidemiologia sugestiva. Antecedentes de diagnóstico e tratamento prévio de brucelose foram referidos por cerca de um quarto dos doentes, facto que sugere que a EB pode surgir no decurso ou após terapêutica antibiótica. No entanto, não possuímos dados relativos à maioria dos diagnósticos e tratamentos realizados previamente (desde três meses até 10 anos antes) e assim, também tendo em conta que se trata de doentes que mantiveram factores de risco para aquisição da infecção, tornou-se impossível interpretar, com rigor, a espondilodicite brucélica como recidiva ou reinfecção. De igual modo, também pela relativa escassez de dados registados e por, com frequência, se tratarem de diagnósticos e tratamentos conduzidos noutras instituições, se optou por não aprofundar a avaliação da questão de outras focalizações brucélicas concomitantes com a espondilodiscite. Apesar de se terem apurado relatos de neurobrucelose $(n=$ $3)$, poliartrite periférica $(n=3)$ e sacroileíte $(n=1)$, os constrangimentos anteriormente notados não permitiram definir correctamente o seu perfil temporal em relação à EB, bem como se se tratavam, ou não, de reinfecções ou recidivas após tratamentos sub-óptimos, razão pela qual estes dados não constam dos resultados deste estudo.

Nos doentes com EB, os sinais e sintomas mais frequentes são a dor local (93-100\%), a febre (60-92\%) e os défices neurológicos (19-31\%). 2,13,14,16 Na nossa amostra, a expressão clínica foi semelhante à descrita nos estudos previamente citados, com excepção da febre que foi menos comum $(46,3 \%)$. O quadro clínico relativamente inespecífico da EB condiciona dificuldades e atrasos no diagnóstico, com consequente aumento da morbilidade. ${ }^{13}$ Vários estudos referem intervalos de tempos entre início das queixas e diagnóstico entre 1 semana e 103 semanas. s, $^{2,13,17} \mathrm{Na}$ nossa série foi registada uma média de cinco meses e meio de sintomatologia prévia ao diagnóstico, com um período máximo de três anos num doente com evolução insidiosa de cervicalgia.

A RMN da coluna é um exame com elevada sensibilidade no diagnóstico precoce da espondilodiscite, permitindo ainda a avaliação dos tecidos moles envolventes e existência de abcessos nessas estruturas..$^{11,18} \mathrm{Na}$ nossa série todos os doentes foram submetidos a um estudo imagioló- 


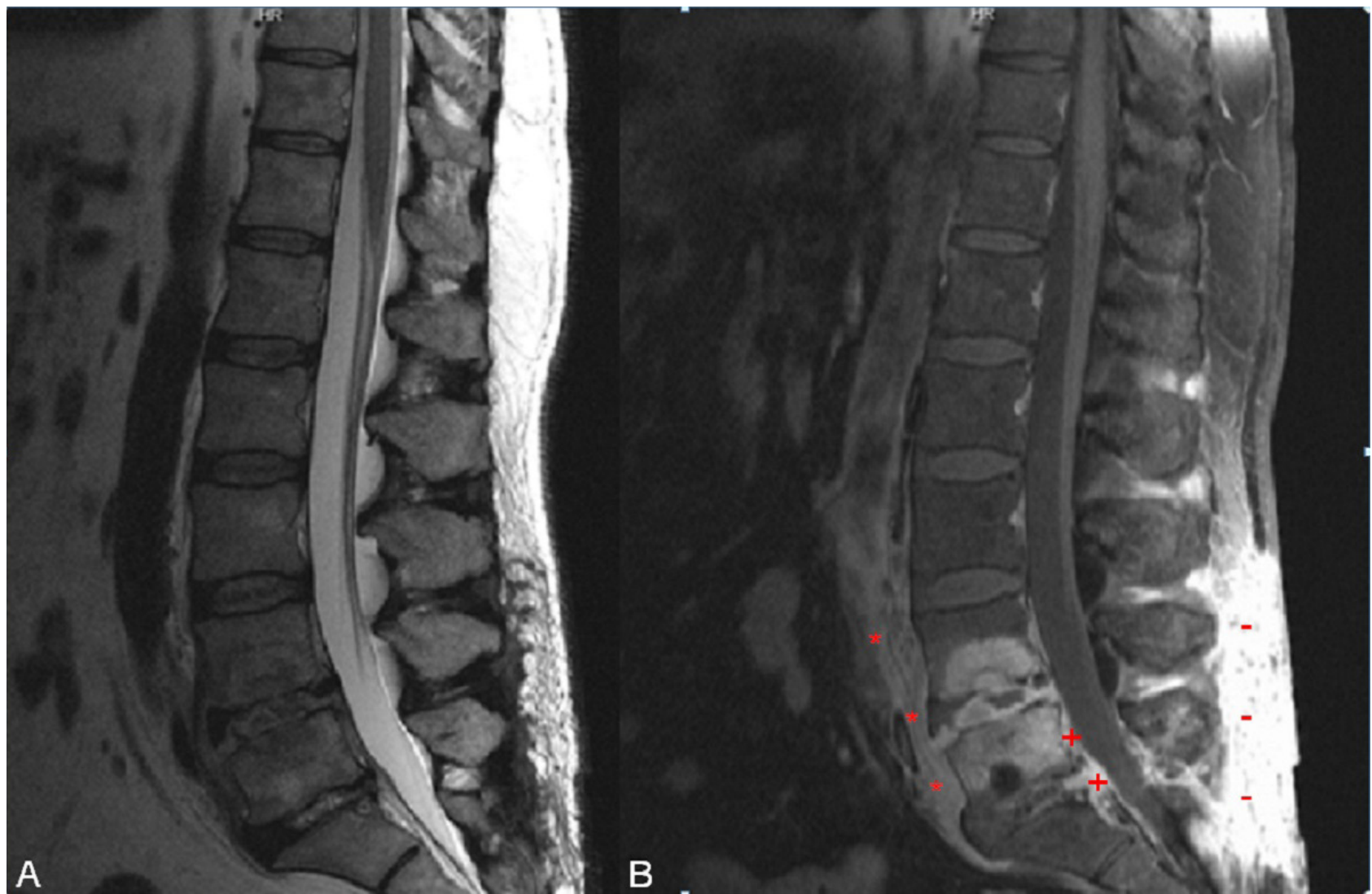

Figura 2 - Ressonância magnética da coluna, plano sagital ponderação T2 (A) e T1 com supressão de gordura após contraste (B) de doente da amostra. Identificam-se alterações do disco intervertebral L4-L5 e plataformas vertebrais adjacentes, em menor grau do disco L5-S1, com extensão pré-vertebral $\left({ }^{*}\right)$, componente epidural contíguo $(+)$ e envolvimento dos tecidos moles posteriores inter-espinhosos (-). Há hipersinal dos discos intervertebrais em T2 (A), com evidente realce discal e dos corpos vertebrais de L4 e de L5 após contraste (B). Nesta imagem o envolvimento relativamente dominante dos corpos vertebrais em relação ao disco vertebral poderá ajudar a distinguir a espondilodiscite brucélica da piogénica e a inexistência de deformidade vertebral evidente da etiologia tuberculosa.

gico da coluna vertebral, com a maioria dos diagnósticos de espondilodiscite a serem feitos por RMN (Fig. 2). Apesar de todos os segmentos da coluna vertebral terem sido afectados, as lesões ao nível do segmento lombar foram as mais frequentes, resultados coincidentes com outras séries. ${ }^{2,3,19}$ Abcessos paravertebrais e epidurais podem surgir associados à $\mathrm{EB}^{14}$, tendo sido observados em $29,6 \%$ dos doentes do nosso estudo.

O diagnóstico definitivo da Brucelose requer o isolamento do agente etiológico no sangue, medula óssea ou outros tecidos ou fluidos biológicos. No entanto, a probabilidade de isolamento do gérmen em cultura de sangue depende da fase da infecção e da técnica de cultura usada, com valores entre $80-90 \%$ em fase aguda e $30-70 \%$ em fase crónica. Nos casos em que não é possível o isolamento da Brucella spp., o diagnóstico baseia-se nos métodos serológicos, uma prova indirecta da infecção. ${ }^{20}$ No entanto em doentes com terapêutica prévia, os títulos serológicos poderão ser baixos ${ }^{12}$ e em casos de doença focalizada alguns estudos reportaram baixa sensibilidade ${ }^{13}$ - deste modo, tal não deverá, caso a suspeita etiológica permaneça, levar à exclusão do diagnóstico, tal como ocorreu em alguns doentes da presente amostra. ${ }^{21}$ No nosso estudo a percentagem de culturas positivas foi inferior à previamente descrita, facto que pode ser explicado, em alguns casos, pelo desconhecimento da suspeita de brucelose por parte do laboratório, o que não permitiu optimizar a metodologia usada para a cultura desta bactéria, e noutros por uso de antibioterapia prévia. Nos isolamentos microbiológicos efectuados a espécie não foi apurada pois, além desse resultado não alterar a conduta terapêutica, esta metodologia não é realizada no nosso laboratório. Assim, títulos elevados de anticorpos específicos (teste de Wright $\geq 1: 160$ ), associados a um quadro clínico, imagiológico e, frequentemente, epidemiológico, compatível, foram o método de diagnóstico mais frequentemente usado, sendo o teste de Rosa de Bengala utilizado como forma rápida de rastreio em todos os casos suspeitos.

O tratamento da brucelose osteoarticular ainda é controverso. Questões como, qual o melhor esquema de antibioterapia, qual a duração do tratamento e qual o papel da cirurgia permanecem sem uma resposta definitiva. ${ }^{22} \mathrm{~A}$ maioria dos estudos sugere uma melhor resposta dos regimes de doxiciclina/aminoglicosídeo (estreptomicina e gentamicina) relativamente aos de doxiciclina/rifampicina, ${ }^{9,13,23}$ que no entanto tem a vantagem de ser uma associação de 
administração por via oral. ${ }^{24}$ Segundo uma meta-análise, que avaliou oito estudos relevantes, os resultados estão relacionados com a duração do tratamento e não com o tipo de regime terapêutico usado. ${ }^{25}$ Os doentes com focalização osteo-articular beneficiam de um tratamento prolongado. Um estudo prospectivo de EB, no qual foi avaliada a eficácia de uma terapêutica superior a seis meses, apresentou taxas de sucesso de $100,0 \%$, sem recidivas, sequelas graves ou mortalidade. ${ }^{22}$ Neste estudo, as associações de doxiciclina com rifampicina ou estreptomicina foram as mais frequentes, sendo os regimes com estreptomicina os mais usados nos últimos anos do estudo. O esquema triplo de antibioterapia foi opção em apenas $7,4 \%$ dos doentes. A duração do tratamento foi variável, com uma média de 4,4 meses de antibioterapia, de acordo com a resposta clínica do doente.

A terapêutica cirúrgica é uma opção pouco frequente no tratamento da EB, estando indicada nos casos de persistência ou progressão de défices neurológicos, instabilidade da coluna vertebral e na ausência de resposta a terapêutica médica. ${ }^{26}$

Apesar de terem sido diagnosticados abcessos paravertebrais e/ou epidurais em $29,6 \%$ doentes e compromisso neurológico em $24,1 \%$, apenas um doente foi submetido a uma intervenção cirúrgica por compromisso neurológico condicionado por um abcesso paravertebral.

$\mathrm{Na}$ nossa amostra, tal como descrito em outras ${ }^{2,14}$ a maioria dos doentes teve uma evolução favorável, não havendo registo de óbitos.

Algumas limitações podem ser identificadas neste estudo, desde logo as decorrentes do facto de se tratar de uma análise retrospectiva de processos clínicos onde a precisão da informação recolhida é limitada pela escassez de dados presentes nos registos. O seguimento dos doentes não foi uniformizado, sendo impossível avaliar com maior rigor a ocorrência de recidivas e sequelas a longo prazo.

\section{CONCLUSÕES}

Este estudo descreve a experiência do nosso serviço na abordagem de doentes com EB, reflectindo as dificuldades e atrasos de diagnóstico de uma patologia com uma clínica inespecífica. Em regiões onde a brucelose é endémica, a EB deve ser considerada no diagnóstico diferencial de doentes com queixas de dor ao nível da coluna vertebral, mesmo na ausência de febre, particularmente quando é referida história de contacto com gado ovino ou caprino, ou consumo de leite ou derivados não pasteurizados. A RMN da coluna vertebral é o método imagiológico com maior sensibilidade para o estudo das focalizações osteo-articulares na brucelose, permitindo um diagnóstico mais precoce e melhor avaliação das estruturas locais. O tratamento antibiótico, a sua duração e a necessidade de uma cirurgia adjuvante deverão ser individualizados com vista a um melhor prognóstico que, nesta série, teve uma evolução favorável na maioria dos casos. Embora o presente estudo não permite tirar a esse respeito conclusões definitivas, até pela heterogeneidade de tratamentos realizados e suas respectivas durações, considera-se que provavelmente o esquema mais adequado para o tratamento desta entidade passará por um regime composto por doxiciclina e aminoglicosídeo durante pelo menos seis meses, reservando-se a associação doxiciclina e rifampicina para os doentes que, por toxicidade ou impossibilidade logística, não o possam realizar. Nos últimos anos não foram diagnosticados casos de $E B$, facto que relacionamos com um melhor controlo da endemia animal.

\section{AGRADECIMENTOS}

Os autores agradecem a Gil Cunha o relato da imagem inserida no artigo.

\section{CONFLITOS DE INTERESSE}

Não existem quaisquer conflitos de interesse.

\section{FONTES DE FINANCIAMENTO}

Não foram utilizados subsídios ou bolsas para a realização deste trabalho.

\section{REFERÊNCIAS}

1. Cottle L, Riordan T. Infectious spondylodiscitis. J Infect. 2008;56:401-12.

2. Colmenero JD, Jimenez-Mejias ME, Sanchez-Lora FJ, Reguera JM, Palomino-Nicas J, Martos F, et al. Pyogenic, tuberculous, and brucellar vertebral osteomyelitis: a descriptive and comparative study of 219 cases. Ann Rheum Dis. 1997;56:709-15.

3. Capelo J, Carragoso A, Albuquerque C, Mocho ML, Canto-Moreira N. Espondilodiscite Infecciosa: o estudo de quarenta e um casos. Acta Reumatol Port. 2007;32:255-62.

4. Pappas G, Papadimitriou P, Akritidis N, Christou L, Tsianos EV. The new global map of human brucellosis. Lancet Infect Dis. 2006;6:91-9.

5. Direcção-Geral da Saúde. Doenças de Declaração Obrigatória 19911995. Lisboa: Direcção de Serviços de Educação e Promoção da Saúde, Divisão de Epidemiologia e Bioestatística; 1997.

6. Direcção-Geral da Saúde. Doenças de Declaração Obrigatória 20042008. Lisboa: Direcção de Serviços de Educação e Promoção da Saúde. Divisão de Epidemiologia e Bioestatística; 2009.

7. Buzgan T, Karahocagil MK, Irmak H, Baran Al, Karsen H, Evirgen O, et al. Clinical manifestations and complications in 1028 cases of brucellosis: a retrospective evaluation and review of the literature. Int $\mathrm{J}$ Infect
Dis. 2010;14:e469-78.

8. Mantur BG, Amarnath SK, Shinde RS. Review of clinical and laboratory features of human brucellosis. Indian J Med Microbiol. 2007;25:188-202.

9. Solera J, Lozano E, Martinez-Alfaro E, Espinosa A, Castillejos ML, Abad L. Brucellar spondylitis: review of 35 cases and literature survey. Clin Infect Dis. 1999;29:1440-9.

10. Colmenero JD, Cisneros JM, Orjuela DL, Pachon J, Garcia-Portales R, Rodriguez-Sampedro F, et al. Clinical course and prognosis of Brucella spondylitis. Infection. 1992;20:38-42.

11. Pourbagher A, Pourbagher MA, Savas L, Turunc T, Demiroglu YZ, Erol I, et al. Epidemiologic, clinical, and imaging findings in brucellosis patients with osteoarticular involvement. AJR Am J Roentgenol. 2006;187:87380.

12. Lopes C, Oliveira J, Malcata L, Pombo V, Da Cunha S, Corte-Real R, et al. Espondilite brucélica. Quatro anos de experiência. Acta Med Port. 1992;5:419-23.

13. Colmenero JD, Ruiz-Mesa JD, Plata A, Bermudez $P$, Martin-Rico $P$, Queipo-Ortuno MI, et al. Clinical findings, therapeutic approach, and outcome of brucellar vertebral osteomyelitis. Clin Infect Dis. 2008;46:426- 
33.

14. Kaptan F, Gulduren HM, Sarsilmaz A, Sucu HK, Ural S, Vardar I, et al. Brucellar spondylodiscitis: comparison of patients with and without abscesses. Rheumatol Int. 2013;33:985-92.

15. Kursun E, Turunc T, Demiroglu Y, Arslan H. Evaluation of four hundred and forty seven brucellosis cases. Intern Med. 2013;52:745-50.

16. Turunc $T$, Demiroglu $Y Z$, Uncu H, Colakoglu S, Arslan H. A comparative analysis of tuberculous, brucellar and pyogenic spontaneous spondylodiscitis patients. J Infect. 2007;55:158-63.

17. Bosilkovski M, Krteva L, Caparoska S, Dimzova M. Osteoarticular involvement in brucellosis: study of 196 cases in the Republic of Macedonia. Croat Med J. 2004;45:727-33.

18. Ozaksoy D, Yucesoy K, Yucesoy M, Kovanlikaya I, Yuce A, Naderi S. Brucellar spondylitis: MRI findings. Eur Spine J. 2001;10:529-33.

19. Santiago T, Rovisco J, Silva J, Pereira da Silva JA. Brucelose osteo-articular um retrato dos últimos 10 anos. Acta Reumatol Port. 2011;36:120-5.

20. Al Dahouk S, Nockler K. Implications of laboratory diagnosis on brucellosis therapy. Expert Rev Anti Infect Ther. 2011;9:833-45.

21. Celik AD, Yulugkural Z, Kilincer C, Hamamcioglu MK, Kuloglu F, Akata
F. Negative serology: could exclude the diagnosis of brucellosis? Rheumatol Int. 2012;32:2547-9.

22. Ioannou S, Karadima D, Pneumaticos S, Athanasiou H, Pontikis J, Zormpala A, et al. Efficacy of prolonged antimicrobial chemotherapy for brucellar spondylodiscitis. Clin Microbiol Infect. 2011;17:756-62.

23. Ariza J, Gudiol F, Pallares R, Viladrich PF, Rufi G, Corredoira J, et al. Treatment of human brucellosis with doxycycline plus rifampin or doxycycline plus streptomycin. A randomized, double-blind study. Ann Intern Med. 1992;117:25-30.

24. Pappas G, Siozopoulou V, Akritidis N, Falagas ME. Doxycycline-rifampicin: physicians' inferior choice in brucellosis or how convenience reigns over science. J Infect. 2007;54:459-62.

25. Pappas G, Seitaridis S, Akritidis N, Tsianos E. Treatment of brucella spondylitis: lessons from an impossible meta-analysis and initial report of efficacy of a fluoroquinolone-containing regimen. Int $\mathrm{J}$ Antimicrob Agents. 2004;24:502-7.

26. Alp E, Doganay M. Current therapeutic strategy in spinal brucellosis. Int $\mathrm{J}$ Infect Dis. 2008;12:573-7. 
Ana LEBRE, Jorge VELEZ, Diana SEIXAS, Eduardo RABADÃO, Joaquim OLIVEIRA, J. SARAIVA DA CUNHA, A. Meliço SILVESTRE

\section{Espondilodiscite Brucélica: Casuística dos Últimos 25 Anos \\ Acta Med Port 2014:27:204-210}

Publicado pela Acta Médica Portuguesa, a Revista Científica da Ordem dos Médicos

Av. Almirante Gago Coutinho, 151

1749-084 Lisboa, Portugal.

Tel: +351218428215

E-mail: submissao@actamedicaportuguesa.com

www.actamedicaportuguesa.com

ISSN:0870-399X | e-ISSN: 1646-0758

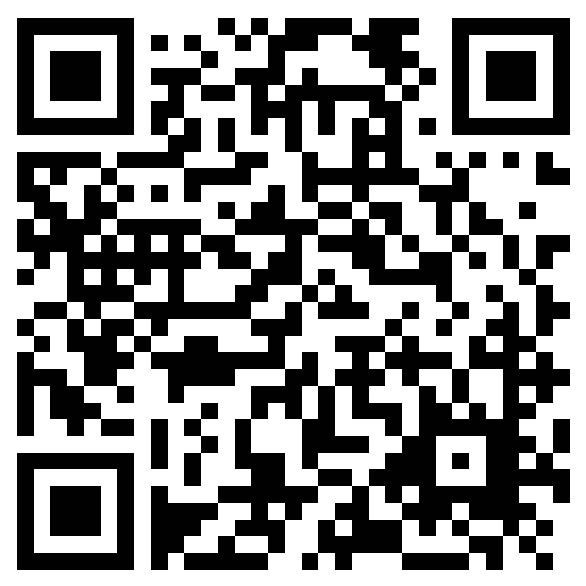

